\title{
Robust Design for Generalized Point Extract CNN with Application in Image Processing
}

\author{
Xiaoliang Zhang ${ }^{1}$, Lequan $\mathrm{Min}^{1}, \mathrm{Ye} \mathrm{Li}^{2}$, Lutao Zhao ${ }^{1, *}$ and $\mathrm{Min} \mathrm{Li}^{1}$ \\ ${ }^{I}$ School of Mathematics and Physics, University of Science and Technology Beijing, Beijing 100083, PR China \\ ${ }^{2}$ University of Science and Technology Beijing, Basic Experimental Center for Natural Science, Beijing 100083, PR \\ China
}

\begin{abstract}
The cellular neural/nonlinear network (CNN) is a powerful tool for image and video signal processing, as well as robotic and biological visions. The designs for $\mathrm{CNN}$ templates are one of the important issues for the practical applications of CNNs. This paper first describes and proves the local rules of the binary Point Extract (PE) CNN introduced by Roska et al., then extends the PE CNN to a gray similar neighborhood pixel remover (SNPR) CNN. The robust design theorem of the SNPR CNN has been established, using a PE CNN and a SNPR processes several images. The results agree with theoretical predictions. In particular, combining the SNPR CNN with median filtering approach is able to remove the salt \& pepper noise in images.
\end{abstract}

Keywords: Cellular neural network, Median filtering approach, Noise, Robust.

\section{INTRODUCTION}

The CNN was first introduced by Chua \& Yang $[1,2]$ in 1988. Its original intention was to find out a structure of neural network that is easier to implement than the Hopfield neural networks [3], which requires to be fully-connected and grows exponentially with the size of the array. Now CNN plays important roles in many fields such as image and video signal processing, robotics and biological visions, and data prediction [3-8].

In an analog cellular neural network, the parameter levers usually have $5 \% \sim 10 \%$ of perturbation [9]. So, the robustness designs for $\mathrm{CNN}$ template parameters are important for the practical applications of CNN. Chua and Dogaru [3,5] have studied the robust designs of a large kind of CNNuncoupled Boolean CNNs, which provide optimal design schemes for CNNs with prescribed tasks. Since then, some robust designs for uncoupled and coupled CNNs have been studied [10-20], which have been used in image processing and pattern recognition.

The rest of this paper has been organized as follows, Section 2.1 describes and proves the Local Rules of the Point Extraction (PE) CNN introduced by Roska et al. [21]. Section 2.2 extends the PE CNN to similar neighborhood pixels remover (SNPR) CNN, and proves a robust theorem for the SNPR. Section 3 gives examples of the application of the CNN. Conclusions have been drawn in Section 4.

*Address correspondence to this author at the School of Information Engineering, School of Mathematics and Physics, University of Science and Technology Beijing, Beijing, 100083, P.R. China; Tel: 13521776678; E-mail: allan_ke@163.com

\section{ROBUSTNESS DESIGN OF CNN}

\subsection{Introduction of $\mathrm{CNN}$}

The standard CNN is composed of a two-dimensional $M$ and $N$ array of cells. Each cell is denoted by $C(i, j)$ where $i=1,2, \mathrm{~L}, M ; j=1,2, \mathrm{~L}, N$. The dynamics of each cell is given by the equation [3]:

$x_{i, j}=-x_{i, j}+\sum_{k=-1}^{1} \sum_{l=-1}^{1} a_{k, l} y_{i+k, j+l}+\sum_{k=-1}^{1} \sum_{l=-1}^{1} b_{k, l} u_{i+k, j+l}+z$

where $u_{i, j}$, and $y_{i, j}$ ae the input, state, and output variables of the cell; $a_{i, j}^{\prime} \mathrm{s}, b_{k, l}^{\prime} \mathrm{s}$ and $z$ are the elements of the $A$ templates and the $B$-templates and threshold, respectively. The output $y_{i, j}$ is the piece-wise linear function given by:

$y_{i+k, j+l}=\frac{1}{2}\left(\left|x_{i+k, j+l}+1\right|-\left|x_{i+k, j+l}-1\right|\right)$

For the Point Extraction (PE) CNN, the standard template has the following form [3]:

$A=\left[\begin{array}{lll}0 & 0 & 0 \\ 0 & 1 & 0 \\ 0 & 0 & 0\end{array}\right], \quad B=\left[\begin{array}{ccc}-1 & -1 & -1 \\ -1 & 1 & -1 \\ -1 & -1 & -1\end{array}\right], Z=-8$

The performances of the PE CNN are described via its Global Task and Local rules:

\section{Global Tasks}

Given: A static binary $P$ 


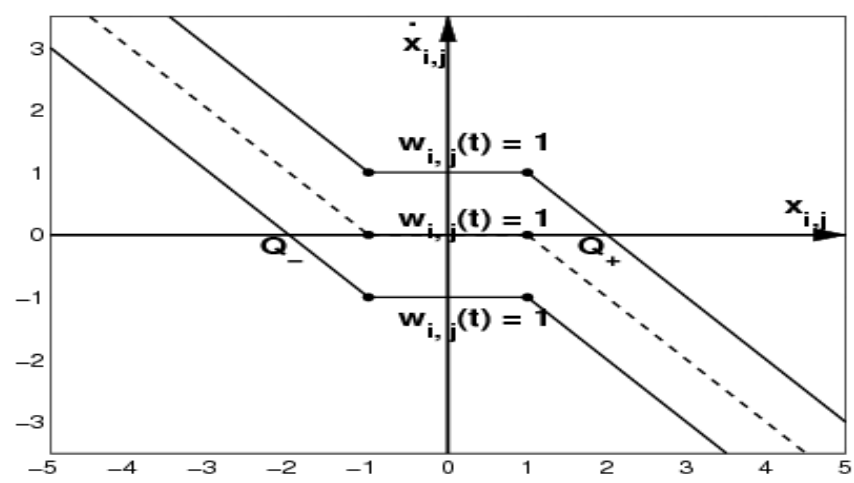

Fig. (1). Dynamic routes of the $\mathrm{CNN}$ where $\mathrm{a}=1$.

Input: $U(t)=P$

Initial State: $X(0)=P$

Output: $Y(t)=Y(\infty)=$ Binary image representing all isolated black pixels in $P$

For processing binary Images, local rules of $\mathrm{CNN}$ can be summarized as follows:

\section{Local Rules}

$u_{i, j}(0) \quad y_{i, j}(\infty)$

White independent of neighbors.

Black if all pixels in the neighbors of the center pixel

$u_{i, j}$ are white.

White if there is at least one black pixel in the $3 \times 3$ neighbors of the center pixel $u_{i, j}$.

Now the following hypothesis has been suggested:

Theorem 1: Assume that the template of the CNN is defined by (2). Then the CNN can implement the Local Rules given above.

Proof. In this case, Equation (1) becomes

$\dot{x}_{i, j}=-x_{i, j}+y_{i, j}+u_{i, j}-\sum_{(k, l) \neq(0,0)} \sum_{k, l \in\{-1,0,1\}} u_{i+k, j+l}-8$

$\dot{x}_{i, j}=-x_{i, j}+y_{i, j}+u_{i, j}-\sum_{(k, l) \neq(0,0)} \sum_{k, l \in\{-1,0,1\}} u_{i+k, j+l}-8$

and denote

$w_{i, j}=u_{i, j}-\sum_{(k, l) \neq(0,0)} \sum_{k, l \in\{-1,0,1\}} u_{i+k, j+l}-8$

From Fig. (1), it follows that:

$y_{i, j}(\infty)= \begin{cases}+1 & w_{i, j}>0 \\ x_{i, j}(0) & w_{i, j}=0 \\ -1 & w_{i, j}<0\end{cases}$
Case 1. If $u_{i, j}=-1$, then

$$
\begin{aligned}
w_{i, j} & =-1-\sum_{(k, l) \neq(0,0)} \sum_{k, l \in\{-1,0,1\}} u_{i+k, j+l}-8 \\
& =-9-\sum_{(k, l) \neq(0,0)} \sum_{k, l \in\{-1,0,1\}} u_{i+k, j+l} \\
& \leq-9+8=-1<0
\end{aligned}
$$

From Equation (3), it can be concluded the Local Rule 1 can be satisfied.

Case 2. If $u_{i, j}=1$ and all pixels in the $3 \times 3$ neighbors of the center pixel $u_{i, j}$ are white, then

$$
w_{i, j}=1-\sum_{(k, l) \neq(0,0)} \sum_{k, l \in\{-1,0,1\}} u_{i+k, j+l}=1+8-8=1>0
$$

From Equation (3), it can be concluded the Local Rule 2 can be satisfied.

Case 3. If $u_{i, j}=1$ and there is at least one black pixel in the $3 \times 3$ neighbors of the center pixel $u_{i, j}$, then

$$
w_{i, j}=1-\sum_{(k, l) \neq(0,0)} \sum_{k, l \in\{-1,0,1\}} u_{i+k, j+l}-8<=1+6-8=-1<0
$$

From Equation (3), it can be concluded the Local Rule 3 can be satisfied.

In summary, when all Local Rules 1-3 can be satisfied, this completes the proof.

The PE CNN can extract the all isolated black pixels, and remove the black pixels with adjacent black pixels. An image processing example of PE CNN is shown in the Fig. (2).

\subsection{SNPR CNN and Robustness Design Theorem}

Let $P$ be a gray scale image with $M \times N$, pixels. Assume $u$ and $u_{1}$ are two adjacent pixels of the image $P . g$ is a given threshold value of similarity degree between two pixels, $u_{1}$ is said to be similar to $u$, if $\left|u_{1}-u\right| \leq g$, otherwise $u_{1}$ is called dissimilar to $u$. For the SNPR CNN, it can remove the pixels with at least $n$ similar nearest neighbors. 


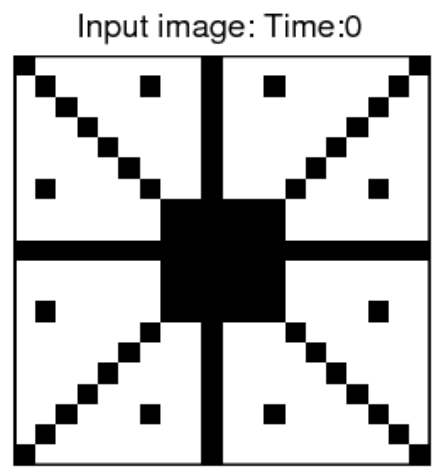

(a)

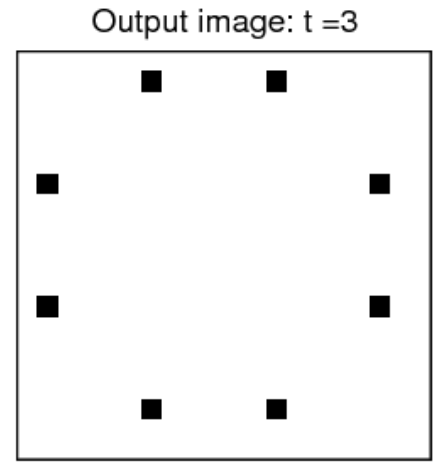

(b)

Fig. (2). An image processing example of PE CNN. (a) The input image, and (b) the output image.

Assume the template of the SNPR CNN has the following form:

$$
A=\left[\begin{array}{lll}
0 & 0 & 0 \\
0 & a & 0 \\
0 & 0 & 0
\end{array}\right], \quad B=\left[\begin{array}{ccc}
b_{-1,-1} & b_{-1,0} & b_{-1,1} \\
b_{0,-1} & b_{0,0} & b_{0,1} \\
b_{1,-1} & b_{1,0} & b_{1,1}
\end{array}\right], \quad Z=z
$$

We will design SNPR CNN such that it satisfies the following Global Tasks and Local Rules:

\section{Global Tasks}

1. Given: A static binary $P$

2. Input: $U(t)=P$

3. Initial State:

$$
\begin{aligned}
x_{i, j}(0)= & \operatorname{sign}\left(\sum_{(k, l) \neq(0,0)} \sum_{k, l \in\{-1,0,1\}} \operatorname{sign}\left(\left|u_{i+k, j+l}-u_{i, j}\right|-g\right)\right. \\
& -8+2 n-0.5)
\end{aligned}
$$

1. Output: $Y(t)=Y(\infty)=$ Binary image where the gray pixel with at least $n$ similar adjacent pixels (the differences of gray less than $g$ ) becomes white, or else becomes black.

\section{Local Rules}

$u_{i, j}(0) \quad y_{i, j}(\infty)$

1. gray white, if there are at least $n$ pixels in the $3 \times 3$ neighbors of the center pixel $u_{i, j}$ satisfy

$\left|u_{i+k, j+l}-u_{i, j}\right| \leq g, k, l \in\{-1,0,1\}$.

2. gray black, if there are at most $n-1$ pixels in the $3 \times 3$ neighbors of the center pixel $u_{i, j}$ satisfy

$\left|u_{i+k, j+l}-u_{i, j}\right| \leq g, k, l \in\{-1,0,1\}$.

In the Initial State, the sign function will be redefined as follows: $\operatorname{sign}(x)= \begin{cases}1 & \text { if } x>0 \\ -1 & \text { if } x \leq 0\end{cases}$

Theorem 2: Assume $a>1$, Let the elements of CNN template be described by (4). Then the CNN can perform the Local Rules, if the following parameter inequalities hold:

$$
\begin{aligned}
& \sum_{k=-1}^{1} \sum_{l=-1}^{1}\left|b_{k, l}\right|+z \geq a-1 \\
& \sum_{k=-1}^{1} \sum_{l=-1}^{1}-\left|b_{k, l}\right|+z \leq 1-a
\end{aligned}
$$

Proof. When $a>1$, From Fig. (3), it follows that:

$$
y_{i, j}(\infty)= \begin{cases}+1 & \text { if } w_{i, j} \geq 1-a, x_{i, j}(0)=1 \\ +1 & \text { if } w_{i, j}<1-a, x_{i, j}(0)=1 \\ -1 & \text { if } w_{i, j} \leq a-1, x_{i, j}(0)=-1 \\ -1 & \text { if } w_{i, j}>a-1, x_{i, j}(0)=-1\end{cases}
$$

Let $p_{1}$ and $p_{g}$ denote, respectively, the total number of the nearest pixels satisfy $\left|u_{i+k, j+l}-u_{i, j}\right| \leq g$ and the nearest pixels satisfy $\left|u_{i+k, j+l}-u_{i, j}\right|>g$.

\section{Case 1.}

When $\quad p_{l} \geq n, \quad x_{i, j}(0)=\operatorname{sign}\left(2 n-2 p_{l}-0.5\right)=-1 \quad$ and $w_{i, j}=\sum_{k=-1}^{1} \sum_{l=-1}^{1} b_{k, l} u_{i+k, j+l}+z$. Then from Equation (7), it follows that the Local Rule 1 is to hold, if the following inequality hold:

$$
w_{i, j}=\sum_{k=-1}^{1} \sum_{l=-1}^{1} b_{k, l} u_{i+k, j+l}+z \leq \sum_{k=-1}^{1} \sum_{l=-1}^{1}\left|b_{k, l}\right|+z \leq a-1
$$

This completes the Local Rule 1. 


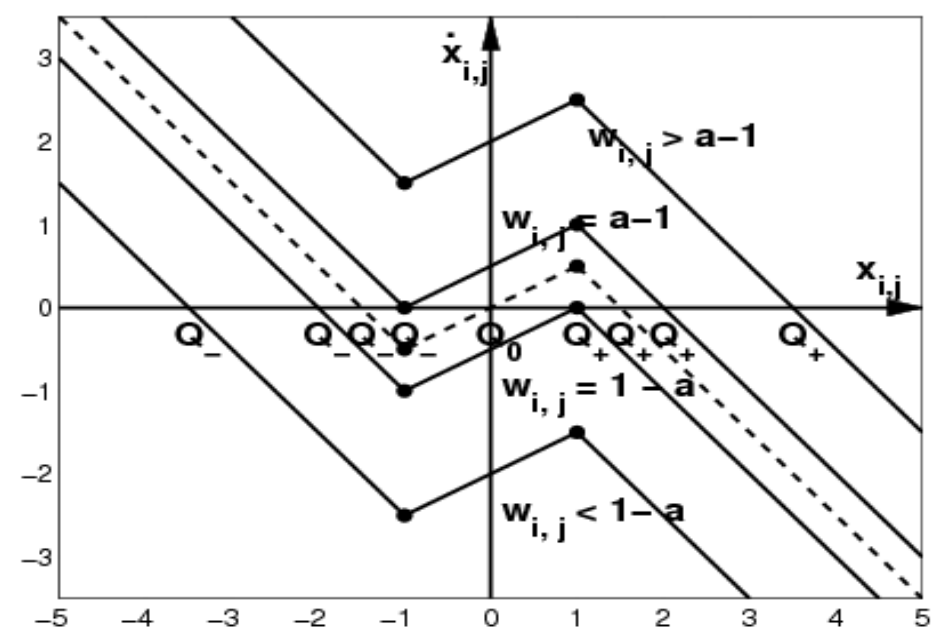

Fig. (3). Dynamic routes of the CNN where $a>1$.

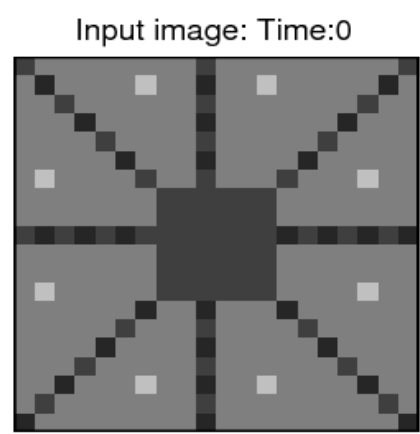

(a)

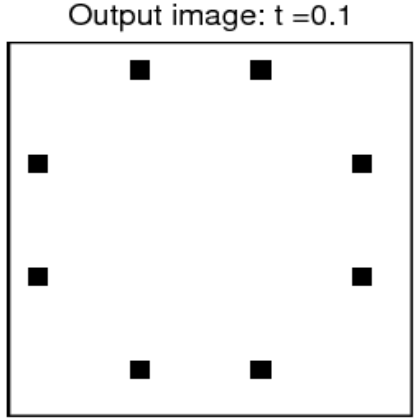

(b)

Fig. (4). An example of the standard SNPR CNN. (a) The input image, and (b) the output image.

\section{Case 2.}

When $p_{l} \leq n-1$ and $x_{i, j}(0)=\operatorname{sign}\left(2 n-2 p_{l}-0.5\right)=1$, Then from Equation (7), it follows that the Local Rule 2 is to hold, if the following inequality hold:

$w_{i, j}=\sum_{k=-1}^{1} \sum_{l=-1}^{1} b_{k, l} u_{i+k, j+l}+z \geq \sum_{k=-1}^{1} \sum_{l=-1}^{1}-\left|b_{k, l}\right|+z \geq 1-a$.

Hence if inequalities (6) hold, then Local Rule 1 and Local Rule 2 both hold. This completes the proof.

\section{EXPERIMENTAL SIMULATIONS}

Firstly, let us use the SNPR CNN with parameters:

$$
A=\left[\begin{array}{lll}
0 & 0 & 0 \\
0 & 9 & 0 \\
0 & 0 & 0
\end{array}\right] \quad, \quad B=\left[\begin{array}{ccc}
-1 & -1 & -1 \\
-1 & 1 & -1 \\
-1 & -1 & -1
\end{array}\right] \quad \text { and }
$$

$Z=0, n=1, g=0.1$ to process a gray image shown in Fig. (4a). The processed image is described as follows: the gray level of the image's background is 0.25 , the gray level of the independent pixels are 0.75 , the other pixels' gray difference with their neighbors' less than 0.1. Observe that the processed result in Fig. (4b) of the image is a binary image such that all the isolated gray pixels whose gray scale difference between it and its nearest neighbors is larger than $g$, have been changed to black ones.

Generally speaking, each pixel of a gray scale image has some similar nearest pixels. While there is no such commonly held property for the pixels which are salt \& pepper noise. So, the SNPR CNN is able to extract the salt \& pepper noise in an image. Then, we can use the median filtering approach to filter them off. The process of removing salt \& pepper noise is described by the following:

1. Extracting the noise: in order to sufficiently extract the noise from an image $P$, the threshold $g$ should not be too big, and $n$ should not be too small. We choose $g=0.1$ and $n=2$. Use the SNPR CNN to extract the salt \& pepper noise. The extracting result is a binary image denoted by $P 1$. 


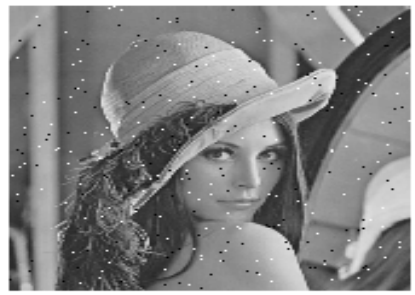

(a)

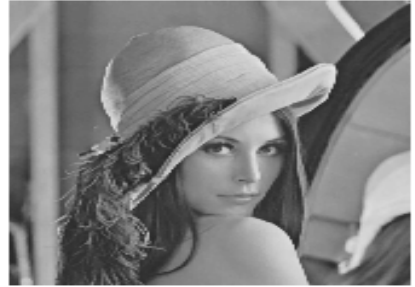

(d)

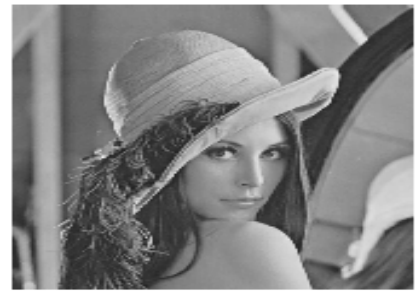

( $\mathrm{g})$

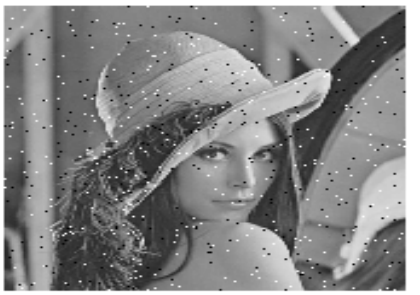

(b)

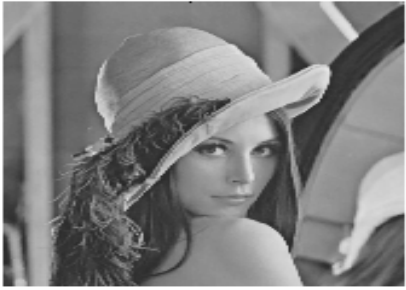

(e)

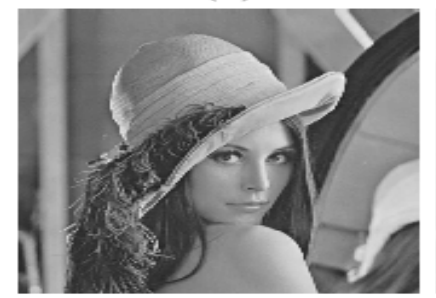

(h)

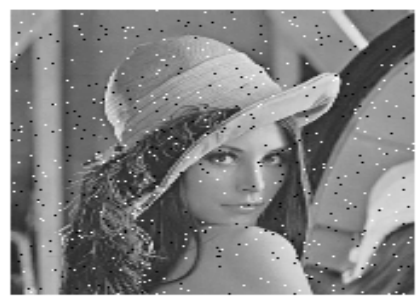

(c)

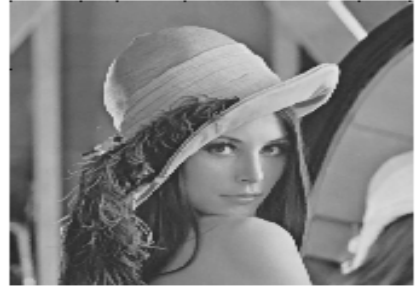

(f)

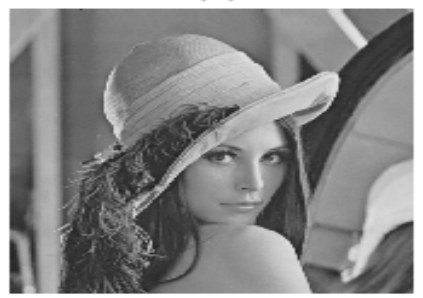

(i)

Fig. (5). Comparison of median filtering and our method. The images shown in the top row are the Lena portraits with different noise intensities. The ones in the middle row are processed images by median filtering approach. The ones in the bottom row are processed images via the SNPR CNN with median filtering approach.

Table 1. Result for Lena with 0.01 noise intensity.

\begin{tabular}{|c|c|c|c|}
\hline Methodslcriterion & PSNR & MSE & MAE \\
\hline \hline Median Filtering & 41.7285 & 4.3675 & 0.8041 \\
\hline Our Method & 55.2463 & 0.1943 & 0.0295 \\
\hline
\end{tabular}

2. Filtering the noise: find all the positions of the white pixels in the image $P 1$. Using median filtering approach to filter the pixels in the image $P$ in the corresponding position. Then, obtain an image without the salt $\&$ pepper noise, denoted by $P 2$.

Now we give examples. The images in the top row of Fig. (5) are the Lena portraits with different noise intensities. Fig. (5)(a) is the Lena portrait with 0.01 noise intensities. Fig. (5)(b) is the Lena portrait with 0.02 noise intensities. Fig. (5)(c) is the Lena portrait with 0.05 noise intensities. The processed Lina images by median filtering approach in matlab are shown in the middle row of Fig. (5). The processed Lina images via the SNPR CNN with median filtering approach are shown in the bottom row of Fig. (5).

Then we use the criterion Peak Signal to Noise Ratio (PSNR) Mean Square Error (MSE) and Mean absolute Error (MAE) to compare median filtering approach with our method. Assume $P$ is an original image, $I$ is the processed im- age, the size of $P$ is $m \times n$. PSNR, MSE and MAE are defined in Equation (8).

$$
\begin{array}{r}
M S E=\frac{\sum_{i=1}^{m} \sum_{j=1}^{n}\left(I_{i, j}-P_{i, j}\right)^{2}}{m \times n} \\
P S N R=10 \times \log \left(\frac{255^{2}}{M S E}\right) \\
M A E=\frac{\sum_{i=1}^{m} \sum_{j=1}^{n}\left|I_{i, j}-P_{i, j}\right|}{m \times n}
\end{array}
$$

It is well-known that the larger the PSNR value, the better the efficacy of reducing noise; the smaller the MSE and MAE values, the better the efficacy of reducing noise. Tables 1-3 listed the comparison data of the median filtering approach and the SNPR CNN with median filtering approach. 
Table 2. Result for Lena with 0.02 noise intensity.

\begin{tabular}{|c|c|c|c|}
\hline Methodslcriterion & PSNR & MSE & MAE \\
\hline \hline Median Filtering & 41.5605 & 4.5398 & 0.8228 \\
\hline Our Method & 53.1350 & 0.3159 & 0.0451 \\
\hline
\end{tabular}

Table 3. Result for Lena with 0.05 noise intensity.

\begin{tabular}{|c|c|c|c|}
\hline Methods $>$ criterion & PSNR & MSE & MAE \\
\hline \hline Median Filtering & 41.1539 & 4.9853 & 0.8822 \\
\hline Our Method & 49.7948 & 0.6817 & 0.0969 \\
\hline
\end{tabular}

It has been observed that the PSNR value of the SNPR CNN with median filtering approach is larger than the one of median filtering approach; the MSE and MAE values of the SNPR CNN with median filtering approach are smaller than the ones of median filtering approach.

\section{CONCLUSION}

The PE CNN is first proposed by Roska et al. in [21] with the aim to extract the isolated black pixels in the binary image. This paper sets up theorem 1 to illustrate what Local Rules of the PE CNN should be satisfied, and proves them. An image processing example illustrates the efficacy of theoretical results in computer simulations.

Then we extend the PE CNN to SNPR CNN via designing its specific initial state defined Equation (5). This technique makes the SNPR CNN to be able to process gray scale image. Robustness Theorem 2 for the SNPR CNN is presented.

Median filtering approach is an effective tool to filter out the salt \& pepper noise in images. This paper combines the SNPR CNN with median filtering approach to process the images with different intensities of salt \& pepper noises. Comparing our approach with the median filtering approach shows that our approach is promising.

\section{CONFLICT OF INTEREST}

The authors confirm that this article content has no conflict of interest.

\section{ACKNOWLEDGEMENTS}

The authors gratefully acknowledge the financial support from Beijing Higher Education Young Elite Teacher Project (YETP0386) and Project 71403014 supported by National Natural Science Foundation of China.

\section{REFERENCES}

[1] L. O. Chua and L. Yang, "Cellular neural networks: Theory", IEEE Transactions on Circuits and Systems, vol. 35, pp. 1257-1272, 1988.
[2] L. O. Chua, and L. Yang, "Cellular neural networks: Applications", IEEE Transactions on Circuits and Systems, vol. 35, pp. 12731290, Oct. 1988.

[3] L. O. Chua, "CNN: Visions of complexity", International Journal of Bifurcation and Chaos, vol. 7, pp. 2219-2425, Jul. 1997.

[4] F. Weblin, T. Roska, and L. O. Chua, "The analogic cellular neural network as bionic eye", International Journal of Circuit Theory and Applications, vol. 23, pp. 541- 569, Nov. 1995.

[5] R. Dogaru, and L. O. Chua, "Universal CNN cells", International Journal of Bifurcation and Chaos, vol. 9, pp. 1-48, Jan. 1999.

[6] L. O. Chua, and T. Roska, "Cellular neural networks and visual computing”, Cambridge University Press, Cambridge, 2002.

[7] P. Arena, A. Basile, M. Bucolo, and L. Fortuna, "Image processing for medical diagnosis using CNN", Nuclear Instruments and Methods in Physics Research Section A: Accelerators, Spectrometers, Detectors and Associated Equipment, vol. 497 pp. 174-178, Jan. 2003.

[8] Ü. A. Sahin, C. Bayat, and O. N. Ucan, "Application of cellular neural network $(\mathrm{CNN})$ to the prediction of missing air pollutant data", Atmospheric Research, vol. 101, pp. 314-326, Jul. 2011.

[9] M. Hänggi, and G. S. Moschytz, "Genetic optimization of cellular neural networks", In: Proceedings IEEE International Conference on Evolutionary Computation, Anchorage, AK, pp. 381-386, 1988.

[10] Y. Su, and L. Min, "Robustness designs of templates of directed overstrike CNNs with applications", Journal of Signal Process, vol. 8 pp. 449-454, Jan. 2004.

[11] J. Liu, and L. Min, "Design for CNN Templates with Performance of Global Connectivity Detection”, Communications in Theoretical Physics, vol. 41, pp. 151-156, 2004.

[12] G. Li, L. Min, and H. Zang, "Color edge detections based on cellular neural network", International Journal of Bifurcation and Chaos, vol. 18, pp. 1231-1242, Apr. 2008.

[13] J. Liu, and L. Min, "Robust designs for gray-scale global connectivity detection CNN template", International Journal of Bifurcation and Chaos, vol. 17, pp. 2827-2838, Jan. 2007.

[14] J. Liu, and L. Min, "Robust designs for tempaltes of directional extraction cellular neural network with applications," International Journal of Pattern Recognition and Artificial Intelligence, vol. 23, pp. 87-100, Feb. 2009.

[15] J. Liu, and L. Min, "Robust design of bipolar wave cellular neural network with applications", International Journal of Model, Identification Control, vol. 11, pp. 99-106, Sep. 2010.

[16] F. Chen, L. Chen, and W. Jin, "Robust Designs of Selected Objects Extraction CNN", In: Proceedings of the Second International Congress on Image and Signal Processing, Hangzhou, pp. 11841186, 2009.

[17] Y. L. Lin, J. G. Hsieh, and J. H. Jeng, "Robust template decomposition without weight restriction for cellular neural networks implementing arbitrary boolean functions using support vector classifiers", Mathematical Problems in Engineering, vol. 2013, pp. 1-9, May 2013.

[18] Q. Zhang, and L. Min, "Dynamic Analysis of Coupled Binary Stripe CNNS", In: Proceeding of the 2011 IEEE International Con- 
ference on Intelligent Computation and Bio-Medical Instrumentation, Wuhan, Hubei, pp. 236-240, 2011.

[19] H. Li, X. Liao, C. Li, H. Huang, and C. Li, "Edge detection of noisy images based on cellular neural networks", Communications in Nonlinear Science and Numerical Simulation, vol. 16, pp. 37463759, Sep. 2011.

[20] S. Deng, Y. Tian, X. Hu, P. Wei, and M. Qin, "Application of new advanced CNN structure with adaptive thresholds to color edge de- tection", Communications in Nonlinear Science and Numerical Simulation, vol. 17, pp. 1637-1648, Sep. 2012.

[21] K. Karacs, G. Y. Cserey, Á. Zarándy, P. Szolgay, C. S. Rekeczky,

L. Kek, V. Szabó, G. Pazienza, and T. Roska, Software Library for Cellular Wave Computing Engines, "Cellular Sensory and Wave Computing Laboratory of the Computer and Automation Research Institute", Hungarian Academy of Sciences and the Jedlik Laboratories of the Pzmny P. Catholic University, 2010.

Received: December 15, 2014

(C) Zhang et al.; Licensee Bentham Open.

This is an open access article licensed under the terms of the Creative Commons Attribution Non-Commercial License (http://creativecommons.org/licenses/by$\mathrm{nc} / 3.0 /$ ) which permits unrestricted, non-commercial use, distribution and reproduction in any medium, provided the work is properly cited. 